International Journal of Wireless Communications and Networking Technologies

Available Online at http://warse.org/IJWCNT/static/pdf/file/ijwent01832019.pdf

https://doi.org/10.30534/ijwcnt/2019/01832019

\title{
Review on Moving Zone Based Clustering in Vehicle-to-Vehicle (V2V) Communication
}

\author{
Jaisy P Jacob ${ }^{1}$, Alphonsa Johny ${ }^{2}$ \\ ${ }^{1}$ APJ Abdul Kalam Technological University, India, jaisypjacob13@gmail.com \\ ${ }^{2}$ APJ Abdul Kalam Technological University, India, alphonsajohny1@ gmail.com
}

\begin{abstract}
Vehicular Ad-hoc Networks (VANETs) allow vehicles to commune among each other and form a huge network with vehicles acting themselves as network nodes. A crucial requirement for the perception of VANET applications is that the availableness of effective and economical routing protocols for message propagation. Safety applications accept exchanging messages disseminated to all or any or a particular portion of vehicles. There are various routing protocols, out of that clustering-based protocols are the foremost optimistic one because they attempt to capture the mobility of VANET nodes in a normal way and also provides comparatively stable clusters. The concept related to moving zone is important. This technique provides a naturalistic clusterbased depiction, in which vehicles are categorized based on their current moving pattern. However, the moving zone system has its own disadvantages like difficulty in maintaining the zone, frequent update of real-time information and so on. Many kinds of research are going under this area. This paper mainly focuses on the mobility based clustering in-order to achieve an adept routing protocol for message dissemination.
\end{abstract}

Key words: Cluster-based Routing Protocols;Intersection; Mobility; VANETs.

\section{INTRODUCTION}

Vehicular Ad-hoc network (VANET) is the most significant research area which is a subdivision of MANET due to low-cost, flexibility, fault tolerance, high sensing fidelity, creating many new and exhilarating applications for remote sensing. So, it has emanated as an emerging weapon for supervising the environment with a wireless sensor which can sense, process and communicate. VANET is a trending technology that considers mobile vehicles as nodes in the network in order to form a mobile network. VANET make every participating automobiles into a wireless router or node. VANET is a self-organized network of vehicles (nodes) on road. Recently, VANETs have accomplished extensive relevancy in varied application domains associated with transportation systems like providing public safety and help, driving improvement, toll assortment, roadside service finders, traffic observation and management, highway internet access safety and efficiency of highway systems.

VANET support two different types of communications: (1) vehicle to vehicle (V2V) and (2) vehicle to infrastructure (V2I) [14] which is depicted in Figure 1[13]. Vehicular communications are established as a part of Intelligent Transportation System (ITS). The desire for V2V communication systems is to offer safety and remove the immoderate traffic collisions. The World Health Organization (WHO) states that road accidents cause approximately 1.2 million deaths annually worldwide, out of which quarter of deaths are caused by injury. So the importance of $\mathrm{V} 2 \mathrm{~V}$ communication is more. In $\mathrm{V} 2 \mathrm{~V}$, Dedicated Short Range Communication (DSRC) enables vehicles to interact among each other through OBU [15]. Each vehicle is fixed with On-Board Units (OBUs), which has omnidirectional antennas, processors, GPS unit, and sensors for communions.

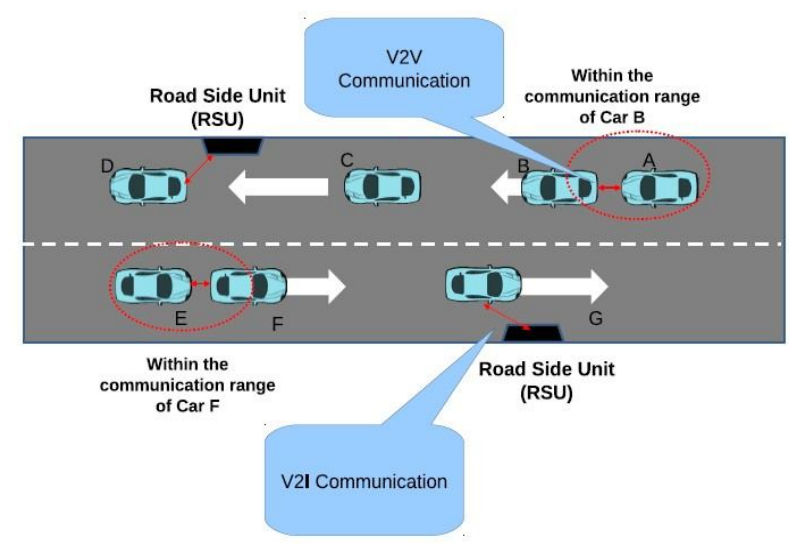

Figure 1: Communication in VANET

Vehicles can also perform V2I communions with roadside infrastructures that are placed in a fixed range depending on the communion range of the roadside devices known as Road Side Units (RSUs). They interact each other across a wireless medium or wired connections. The V2I communions can be expanded since RSUs can connected to a network. The V2V communion is used to transmit some sort of emergency or real-time information such as an accident or road traffic information so that the following 
Jaisy P Jacob et al., International Journal of Wireless Communications and Network Technologies, 8(3), April - May 2019,1 - 7

vehicles can move to the alternative routes to prevent traffic congestions. VANETs allows emergency real-time applications and also deals with life-critical information. For that they should follow the security requirements such as privacy, confidentiality, integrity, and non-repudiation to provide secured communications against attackers and malicious nodes.

The unique traits of VANET include features such as high dynamic topology, frequent dis- connected network, mobility modeling and prediction, communication environment, unlimited transmission power, hard delay constraints, interaction with On-board sensors, higher computational capability, rapidly changing network topology, potentially limitless network size and so on. In the development of smart cities across the world, VANET plays a decisive role for an amended path between source and destination. The VANET architecture is an infrastructure-less network. It eases vehicles to pass information about safety using V2V or V2I communication. The first system that integrates this technology was the fire vehicles and police to communicate among each other for the safety purposes.

\section{CLUSTERING AND MOBILITY}

In order to diffuse information among vehicles, an effective routing protocol must be used. A routing protocol shows how vehicles (nodes) communicate with among others and it also distributes information among them which enables them to find routes between any two nodes.It shares information among one-hop neighbors, and then along the network. This way each node between the initial and final destination get information about its communication path.The specific features of routing protocol embody the way during which they avoid loops, the choice of most well-liked routes, data regarding the hop prices, the time needed to succeed in therouting confluence, their extensibility, and other factors.In VANET, routing protocols can be divided as manifest in Figure 2.

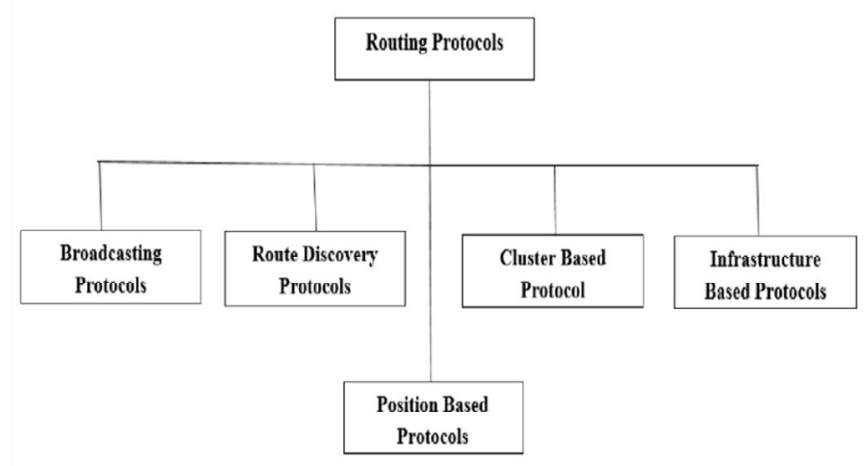

Figure 2: Types of routing protocols in VANET

The broadcasting protocols lie on massive message diffusion, and which may cause communion overhead and message congestion in the network. The Route discovery protocol discovers the path before sending a message and not possible within the given time constraints. In positionbased protocol, nodes are supposed to pass messages to nearest neighbour until destination. Here each vehicle maintains information of the nearest neighbour, so the overall communication cost will be high. In clustering based routing protocol, only $\mathrm{CH}$ keeps information about the neighbouring vehicles whereas the infrastructure based protocol relies heavily on RSU and thus not widely available. Out of these protocols, the cluster-based routing protocol is the most important as they consider the mobility of nodes (vehicles) in a naturalistic way and provide comparably stable networks for communication.

VANET is a subset of MANET. Compared to MANETs, VANETs have some special characteristics as mentioned below. The vehicles in the network have high mobility. So the topography of the network changes very frequently. The quality of the wireless channel is unstable effected by various factors, such as roadside infrastructure, road traffic, vehicle velocity and so on. GPS and digital map provide precise position and precision clock information for the node to plan paths and use its own position information to achieve clock synchronization. Road static shape makes the vehicle movement restricted and vehicle track generally is predictable.

Though many challenges lied in VANET, clustering is an effective way to alleviate those problems. Various vehicles with similar moving features like velocity, direction, speed, location etc., are combined together constructs a cluster in the network. To direct communication among numerous nodes within a cluster or with other clusters, a $\mathrm{CH}$ is needed. Cluster head acts as a coordinator which is used as a channel for data transfer between the nodes. If the packet to be sent is in the same cluster, then it is done by using the direct path.But if the terminal node is not in the same cluster, then $\mathrm{CH}$ will create a virtual network infrastructure which provides scalability. The stability of clusters depends on the election of cluster heads. Different algorithms are used for the election of the cluster head. Stability of a cluster can be estimated based on the following parameters such as the time the cluster head remains the same, time the cluster member remains within their cluster and time the different cluster heads changes.

Since VANET has dynamic topology, the mobility of vehicles within the cluster is to be considered. The mobility metrics of a vehicle which includes the vehicle's speed, current location, the direction which is obtained from GPS. Mobility can be managed either based on location or based on message dissemination path. The following section includes a literature survey on mobility based clustering routing protocol.

\section{LITERATURE REVIEW}

This section divides into two which comprises of various algorithms and methods for the election of a cluster head and also routing algorithms for message dissemination based on the mobility based clustering and intersection concepts.

\subsection{Mobility Based Clustering Routing Protocol}

A clustering scheme based on neighbour vehicle's mobility is introduced by authors in which the $\mathrm{CH}$ is elected based on the neighbour vehicle mobility value [1]. Upon registering vehicles to a network, each vehicle gets a unique id to identify them. All the vehicles in the network will also have 
a neighbour vehicle table (NVT). The parameters in NVT are vehicle ID and neighbour vehicle mobility value (NVMV). To calculate NVMV, the sum of neighbour vehicles entering into and leaving from its coverage area. The NVT get updated whenever it receives a HELLO message. After calculating the mobility value, every vehicle sends HELLO message which includes vehicle id, cluster head id, NVMV, HELLO message time period. Now, the vehicle compares its own mobility value with neighbour vehicles. The vehicle with the lowest NVMV will be elected as the cluster head and thus fixes the remaining vehicle as the cluster members.

The routing scheme mentioned in [2] is for stable rebroadcast nodes election and discovering routes for reliable data transmission. In this, the authors select the rebroadcast nodes based on nearby vehicles with similar velocity (similarity in moving) with the initial vehicle. Here, they assume that all node has a vehicle persistence score (VPS) table. A vehicle with high persistence score is selected as the rebroadcast node, which will remain longer in the inter-vehicle communion path. To reduce the rebroadcast node, a donut-like area is defined to select relay nodes to decrease the network traffic and route hop count. A persistence score is derived. There are two phases and they are neighbour information phase and route discovery phase. In the neighbour information phase, a HELLO message is initially send by each vehicle to its next hop vehicles. There is a neighbour list which contains neighbour vehicle id and neighbour vehicle expiration time. The neighbour list and VPS table get updated upon receiving each HELLO message. The entries into the VPS table are neighbour vehicle id, the position from GPS, the distance between the vehicles and its neighbour, type of neighbour vehicle, VPS. The VPS value is initially assigned as 1 when it first acquires the hello message and it is increased by 1 after reception of HELLO messages. We can fix a maximum value for VPS. In the Route discovery phase, after getting the neighbour information, remove those nodes that are not located between the initial and final nodes. Arrange the remaining neighbours and send the information. Here, we can fix the maximum count of rebroadcast nodes.

A new technique [3] was introduced by authors to identify the very nearest neighbours and select optimal cluster head using mobility metrics. The mobility metrics include speed, location, and direction of a vehicle obtained from GPS. This algorithm generates one hop clusters that are overlapped to form a backbone that allows automobiles to commute. This backbone is used to disseminate neighbor information. Each vehicle has a fixed range of broadcasting. But the main issue in this scheme is it is costly and complex for each node to maintain a neighbour vehicle table. And also, all vehicles in the network cannot distribute information to all other vehicles in the network due to scalability. So the zone of interest (ZOI) concept is introduced. It is to increase the communion range of vehicles. Every node only maintains a neighbour table for nodes within its ZOI and can discard other information receiving from vehicles out of its ZOI. The radius of ZOI of a vehicle can be twice that of the transmission range of that vehicle.
The authors introduced an algorithm based on the destination positions [4]. This algorithm considers the current location, respective destination and terminal destination of vehicles to arrange clusters. $\mathrm{CH}$ keeps a parameter table that stores the vehicle's id, $\mathrm{CH}$ id, current location ( $\mathrm{x}, \mathrm{y})$ coordinates, speed, relative destination and final destination in $(\mathrm{x}, \mathrm{y})$ coordinate. All vehicles conserve a table which stores previous information related to speed, location as $(\mathrm{x}, \mathrm{y})$ coordinates, relative and final destination $(\mathrm{x}, \mathrm{y})$. When a vehicle ' $\mathrm{V}$ ' wants to perceive a $\mathrm{CH}$, it sends affiliation message to all its neighbours, if no reply then begins the cluster formation process. The initial step is to interchange HELLO messages to its one hops and gathers information regarded to the parameter table. In the next step, V computes relative values using the parameter table.

A new dynamic mobility based and stability based clustering for civic city scenario is introduced [5]. It depends on onehop clusters. It considers moving direction of vehicles, link lifetime estimation and relative position. $\mathrm{CH}$ is selected from the centre of the cluster in order to maintain stability. And it also fixes the capacity of the cluster. The length or capacity of the cluster must be minimal than double that of $\mathrm{CH}^{\prime} \mathrm{s}$ transmission space. In this, two gateway nodes are at the brim of the cluster out of which first is moving ahead and the other is at the brim of the cluster. Vehicles on the brim of the of the CH's transmission range does not have much stability which may cause $\mathrm{CM}$ disconnection or $\mathrm{CM}$ re-clustering. So a concept called safe distance threshold (Dt) is introduced. (Dt) should be smaller than vehicles transmission range. Vehicles within (Dt) transmission area of the $\mathrm{CH}$ have more stable links with their $\mathrm{CH}$. So, the capacity of cluster L can be expressed as, $\mathrm{L}<=2$ (Dt). This algorithm is well suited with roads having two lanes. This gives higher cluster stability in a high dynamic scenarios. (Dt) has portable effects on both $\mathrm{CH}$ and $\mathrm{CM}$ duration. With large vehicle velocity, some cluster members may get out of the cluster and also has chance to become isolated. A new group of vehicles will be formed if isolated vehicles cannot connect to the existing clusters, which will increases the count of clusters. So, in this system maximal lane speed is fixed.

The authors proposed a distributed clustering algorithm that creates multi-hop clusters. DCEV creates non-overlapping clusters of vehicles with flexible size based on their mobility features. It uses D-hop clustering scheme for cluster formation. D-hop clustering creates clusters in a way that vehicle is atmost $\mathrm{D}$-hops away from the cluster head. Cluster head is selected by each vehicle within its D-hop distance. The mean relative mobility value of the identified route is calculated by each vehicle. Since there is no cluster overlapping, it offers flexible clusters. There are two phases in this algorithm. The first step is the cluster formation and the next step is the $\mathrm{CH}$ selection [6].Beacon messages are send to all neighbours by every vehicles. Firstly relative mobility value is calculated by every vehicle with its onehop neighbouring vehicles. The relative mobility is calculated with its neighbouring vehicle depending on their location and speed. Information about the neighbour vehicle is attained from the received beacon messages. The next step is that each vehicle has to find the routes compromising up 
to D-hops. The end-to-end relative mobility stands for the mean value of relative mobilities on each route. Select the route with the least mobility value. Find the vehicle which has highest degree of connectivity from the final selected route. If there is multiple nodes with the same connectivity, then select the node that has a minimal deviation from the average speed of vehicles on that route. Since each vehicle needs the speed of the nodes in the final selected path and also relative mobility, a beacon is piggybacked among vehicles.

The authors introduced a beacon based clustering algorithm to increase the cluster life time in VANET. It measures the criteria for cluster re-organization. It also prevents overeager re-organization of clusters upon an accidental contact. That is when two CHs get into each other, before going for reorganization it will wait for a certain period of time. This algorithm is used to decide which cluster head should retain their roles after overlapping [7].

Cluster-based routing is a combination of both static and dynamic clustering. Static clusters are built around static (non-movable) sources located at streets, road signals and so on whereas moving objects are the dynamic sources. Vehicles mobility determines the structure of the cluster. Vehicles are classified into two different ways either by those vehicles which are in the transmission/communion range of dynamic sources or those vehicles which are in the range of static sources. By doing so, re-affiliation and reclustering can be decreased naturally. The authors introduced a method which is used to control the vehicular traffic. A location-based multipath algorithm is introduced to send the real-time updated information and maintain long link time limit which is considered to increase the data delivery ratio [8]. This algorithm works well with vehicles having variable mobility. And also this method minimizes the re-clustering process and creates a number of stable clusters.

\subsection{Intersection Based Routing Protocol}

Intersection based routing has little overhead as compared to direct full path routing. It is not essential to consider the route discovery messages and packets carry the location of destination vehicle and next-intersection only. At each intersection, the routing accord is done sequentially based on the evaluation of adjusting roads. Following subsections include various techniques considering the concept of the intersection.

In VANETs, the carry and forward strategy is used in order to overwhelm the irregular distribution of automobiles in the network. The authors proposed an intersection based routing (IBR) protocol which depends on the direction of packet transmission and moving direction of automobiles [9]. This algorithm is used to determine the least delay in the propagation path. The IBR protocol determines the end-toend delay including carrying delay and the forwarding delay. If the intensity of the segment of road is compact, this algorithm counts only the forwarding delay. The algorithm computes end-to-end delay which is the total of carrying delay and forwarding delay, in the sparse traffic road segment. Here, every vehicle is having a GPS and navigation system. And also, each automobile has a road segment table which consists of current road segment ID, the number of automobiles in that road segment and recording time. From the location server, the destination position and next road ID can be obtained. The sender vehicle knows the terminal vehicle's position and movement direction, with this information a route planning path is determined. If the packet reaches an intersection in the routing path and this intersection is not the destination intersection, this IBR algorithm will find a temporary path which has a minimum delay. If the delay of a temporary path is smaller than the actual route selected, the original path is replaced by the temporary path and the packets routes along the new path. Otherwise the packet is routed along the actual path. When the packet arrives at the intersection, a copy of the packet is kept. One copy of the packet is sent through the current road and the other packet is sent along the next road. Thus packet loss can be avoided at the intersection.

Piggybacking helped many-to-many (M2M) communion with efficient vehicle election for enhanced performance in VANETs. The authors proposed a piggybacking concept along with M2M [10]. Here, roads with many lanes and crossings are considered. The whole road is partitioned into various sections of fixed size 'l'. In the middle of the cell, an RSU is placed. M2M communications are decoded utilizing Code Division Multiple Access (CDMA). In this, GPS was used to attain the current location of the vehicles. GPS aids in synchronizing time between vehicles that communicate among all vehicles in the cells are synchronized. There are two phases in this M2M communication. First is the Setup Phase, here the vehicles have to discover their neighbours and then build a group. Second is the Communication Phase, here the vehicles within a range can be communicated. Piggybacking of information can be done by keeping the updated information about other vehicles and its own. Piggybacking will improve the quality of performance in M2M communication and also enhance the average packet delivery ratio (PDR), average end-to-end delay. In order to find average PDR suppose that there are ' $\mathrm{N}$ ' vehicles in the scenario, every road is partitioned into ' $\mathrm{C}$ ' cells and RSUs are kept after every fixed number of cells (k). Between the successive arrivals of packets at each vehicle uses exponential distribution. The average end-to-end delay can be defined as the total amount of time required for the packet to successfully deliver to a vehicle. In M2M communication, a packet will stay in the vehicle's buffer, until it enters the communion range and the packet is chosen. Vehicles are 
Jaisy P Jacob et al., International Journal of Wireless Communications and Network Technologies, 8(3), April - May 2019, 1 - 7

Table 1: Comparison of Surveyed Papers

\begin{tabular}{|c|c|c|}
\hline $\begin{array}{l}\text { Serial } \\
\text { No. }\end{array}$ & Title of Paper & Technique \\
\hline 1 & $\begin{array}{l}\text { Neighbor Mobility-based Clustering Scheme for Vehicular } \\
\text { Ad Hoc Networks }\end{array}$ & $>$ Based on neighbour vehicles mobility value. \\
\hline 2 & $\begin{array}{l}\text { A Reliable Routing Scheme Based on Vehicle Moving } \\
\text { Similarity for VANETs }\end{array}$ & $>$ Based on neighbour vehicles VPS value. \\
\hline 3 & $\begin{array}{l}\text { Robust mobility adaptive clustering scheme with support for } \\
\text { geographic routing for vehicular ad hoc networks }\end{array}$ & $\begin{array}{l}>\text { To identify 1-hop neighbours and select } \\
\text { optimal CH. } \\
>\text { ZOI concept is introduced. }\end{array}$ \\
\hline 4 & $\begin{array}{l}\text { An Adaptable Mobility-Aware Clustering Algorithm in } \\
\text { Vehicular Networks }\end{array}$ & Based on destination vehicle. \\
\hline 5 & $\begin{array}{l}\text { A mobility-based scheme for dynamic clustering in } \\
\text { vehicular } \\
\text { ad-hoc networks(VANETs) }\end{array}$ & $>$ Based on 1-hop clusters. \\
\hline 6 & $\begin{array}{l}\text { DCEV : A Distributed Cluster Formation for VANET Based } \\
\text { on End-to-End Relative Mobility }\end{array}$ & Based on D-hop clusters. \\
\hline 7 & $\begin{array}{l}\text { A New Aggregate Local Mobility(ALM) Clustering } \\
\text { Algorithm } \\
\text { for VANETs }\end{array}$ & $\begin{array}{l}\text { Beacon based clustering to increase cluster } \\
\text { lifetime. }\end{array}$ \\
\hline 8 & $\begin{array}{l}\text { A novel cluster based routing algorithm for hybrid mobility } \\
\text { model in VANET }\end{array}$ & $>$ To maintain long link duration. \\
\hline 9 & Intersection-Based Routing Protocol for VANET & $\begin{array}{l}\text { To find minimum delay routing path. } \\
\text { Uses carry-and-forward strategy. } \\
\text { Packet routing at intersection depends on the } \\
\text { moving direction of the next vehicle. }\end{array}$ \\
\hline 10 & $\begin{array}{l}\text { Piggybacking assisted many-to-many communication with } \\
\text { efficient vehicle selection for improved performance in } \\
\text { vehicular ad hoc networks }\end{array}$ & $\begin{array}{l}>\text { Offers M2M communication to improve } \\
\text { performance of VANET. } \\
>\quad \begin{array}{l}\text { To improve average PDR and End-to End } \\
\text { delay. }\end{array} \\
>\quad \begin{array}{l}\text { Each road divided into equal number of cells } \\
\text { with same number of vehicles. }\end{array}\end{array}$ \\
\hline 11 & $\begin{array}{l}\text { Lightweight intersection-based traffic aware routing in } \\
\text { Urban } \\
\text { vehicular networks }\end{array}$ & $\begin{array}{l}\text { To reduce network overhead while } \\
\text { preserving the accuracy of the measurement. } \\
\text { Supports multi-hop communication. }\end{array}$ \\
\hline 12 & $\begin{array}{l}\text { MoZo: A Moving Zone Based Routing Protocol Using Pure } \\
\text { V2V Communication in VANETs }\end{array}$ & $\begin{array}{l}\text { Offers stable moving zone based clustering. } \\
\text { Clustering should be done all the time while } \\
\text { vehicles are moving. }\end{array}$ \\
\hline
\end{tabular}


randomly chosen from the communication range of RSUs. A huge number of packets can be disseminated from a vehicle at a single time compared to those without piggybacking. Because, for a vehicle the time spent between two successive transmissions is independent of each other. Once it reaches the RSU, it has to wait in its buffer until it reaches the header of the buffer chosen for communication.

One of the major characteristics of VANETs is the many-tomany communication which allows $\mathrm{V} 2 \mathrm{~V}$ communications. The authors presented two novel algorithms to decrease the network overhead formed from the traffic information measurement process while maintaining the accuracy. Based on this, the routing path is selected depending on directional density, distance towards the target and the point road network connectivity. It supports multihop communication [11].This algorithm works in two steps as follows. The initial step is the Real-Time Traffic \& Network Status Measurement (RTNSM).

This is used for evaluation of road, which is continuously applied in each road to be provided at every intersection. Second is the Data Packet Routing. In this phase, the result of RTNSM is utilized to make decisions at intersections. The

Enhanced Validity Period Calculation (EVPC) algorithm works in the following steps. First, for every neighbour vehicle in the neighbour table, the prediction of current position is based on its mobility information. The neighbour vehicles that are still inside the transmission space and belongs to the same road, a link lifetime (LLT) is calculated. Inter-vehicle communication stability factor (ICS) and validity period (VP) is also calculated (which depends on LLT and ICS). This VP is compared with the received collector packet's validity period value (VPV) field. When a collector packet arrives the evaluated road endpoint, its validity period field will have the minimal calculated VP value amid all the forwarded vehicles on the road.

To utilize the distinctive characteristics of VANET nodes, the authors introduced the concept of moving zone-based architecture which was the first study based on moving-zone architecture where vehicles combine together to create dynamic moving zones that allows information dissemination. The proposed novel approach combines moving object modelling and indexing techniques into VANET routing protocols [12]. This system consists of multiple zones. A CH is chosen zone or cluster. Clustering should be done all the time while the vehicles are moving. Vehicles with less speed deviation and larger travel time will be elected as cluster head. One advantage of clustering is that it is used for message authentication purpose.

The MoZo routing protocol is as follows. The sender vehicle sends the message along with ID of the sender, Message,

Destination location. The captain vehicle initially checks the terminal point is in the identical cluster or not. If it is within the zone, the vehicle can directly communicate. Otherwise, the captain vehicle looks its member vehicles in the nearest moving cluster and forwards a message to that selected vehicle. The captain vehicle uses Dijkstra's algorithm in order to find out the optimum distance between the sender and receiver. It also performs a query algorithm to discover the good propagation node. By using the expected location and desired moving direction, the captain vehicle generates the query key. This will be considered as the key for the virtual vehicle. After finding the leaf node, this virtual insertion will terminate. The resulting leaf node contains a vehicle with a similar key to the virtual vehicle. Otherwise, candidate vehicles will be searched. Captain vehicle will wait for a duration of time and then again search for the candidate vehicle. A maximum of three attempts will be made to send the message. The selected propagation vehicle is responsible for sending a message to the vehicle to the nearby moving zone. All vehicles in the zone keep a record of captain vehicle which replied to HELLO message. This method greatly minimizes communion overhead and improves the accuracy of the message distribution rate compared to other existing methods.

The Table 1 shows the comparison of various papers reviewed and also the technique they used for clustering and $\mathrm{CH}$ selection.

\section{CONCLUSION}

Vehicular Ad-hoc Network is the ensuing transportation system where vehicles commune among each other is a difficult task because of the scattered distribution of nodes. Clustering avoids these problems by teaming up the topographical adjacent vehicles to a cluster. Vehicle clustering can crucially improve the firmness of the system, but intense mobility of vehicles in the cluster influence the stability of the network by frequently altering the network topology. To create a more stable system, formation of cluster and selection of $\mathrm{CH}$ should be considered for the portability of the vehicles. Such a moving cluster creates a secure and stable routing among the network of vehicles. In this paper, we considered the contour of the VANET that defines the communion system which also includes various routing methods, choice of cluster head, and so on.

\section{REFERENCES}

1. Jung-Hyok Kwon, Cheolhee Kwon, Eui-Jik Kim, Neighbor Mobility-based Clustering Scheme for Vehicular Ad Hoc Networks, in International Conference on Platform Technology and Service, 2015.

2. Min-Hsuan Wei, Kuochen Wang, Yi-Ling Hsieh, A Reliable Routing Scheme Based on Vehicle Moving Similarity for VANETs, in IEEE Region 10 Conference , 2011. https://doi.org/10.1109/TENCON.2011.6129139

3. R.T. Goonewardene, F.H. Ali E. Stipidis, Robust mobility adaptive clustering scheme with support for geographic routing for vehicular ad hoc networks, The Institution of Engineering and Technology, 2009. https://doi.org/10.1049/iet-its:20070052 
Jaisy P Jacob et al., International Journal of Wireless Communications and Network Technologies, 8(3), April - May 2019,1 - 7

4. Mildred M. Caballeros Morales, Choong Seon Hong, Young-Cheol Bang, An Adaptable Mobility-Aware Clustering Algorithm in Vehicular Networks, 13th Asia-Pacific Network Operations and Management Symposium, 2011.

5. MengyingRena, LyesKhoukhia, HoudaLabiodb, JunZhangb, VroniqueVquec, A mobilitybased scheme for dynamic clustering in vehicular ad-hoc networks (VANETs), 2016.

6. Meysam Azizian, Soumaya Cherkaoui, Abdelhakim Senhaji Hafid, DCEV : A Distributed Cluster Formation for VANET Based on Endto-End Realtive Mobility, International Wireless Communications and Mobile Computing Conference (IWCMC), 2016.

https://doi.org/10.1109/IWCMC.2016.7577072

7. Evandro Souza, Ioanis Nikolaidis,Pawel Gburzynski, New Aggregate Local Mobility(ALM) Clustering Algorithm for VANET's, IEEE Communications Society subject matter experts for publication in the IEEE ICC, 2010.

https://doi.org/10.1109/ICC.2010.5501789

8. D. Rajini Girinath, S. Selvan, A novel cluster based routing algorithm for hybrid mobility model in VANET, International Journal of Computer Applications Volume 1- No. 15, 2010. https://doi.org/10.5120/326-495

9. Li-Der Chou, Jyun-Yan Yang, Ying-Cheng Hsieh and Chi-Feng Tung, IntersectionBased Routing Protocol for VANET, Second International Conference on Ubiquitous and Future Networks (ICUFN), 2010.

10. Moumita Patra, C.Siva Ram Murthy, Piggybacking assisted many-to-many communication with efficient vehicle selection for improved performance in vehicular ad hoc networks, Volume 108, Pages 223-232, 2016. https://doi.org/10.1016/j.comnet.2016.08.013

11. Tasneem Darwish, Kamalrulnizam Abu Bakar; Lightweight intersection-based traffic aware routing in Urban vehicular networks, Computer Communications 000 (2016) 116, 2016.

12. Dan Lin, Jian Kang, Anna Squicciarini, Yingjie Wu, Sashi Gurung, and Ozan Tonguz, MoZo: A Moving Zone Based Routing Protocol Using Pure V2V Communication in VANETs, IEEE Transactions on Mobile Computing, 2016.

13. Sabihur Rehman, M. Arif Khan, Tanveer A. Zia, Lihong Zheng; Vehicular Ad-Hoc Networks (VANETs) - An Overview aChallenges, Journal of Wireless Networking and Communications, 2013.

14. Felipe Cunha, Leandro Villas, Azzedine Boukerche, Guilherme Maia, Q1 Aline Viana, RaquelA.F. Mini, AntonioA.F. Loureiro, Data communication in VANETs: Survey, applications and challenges ,inComputer Science [cs] / Networking and Internet Architecture [cs.NI],Ad Hoc Networks, Elsevier, 2016, 44 (C), pp.90-10,2016.

15. https://en.wikipedia.org/wiki/Vehicular\$_\$commun ication\$_\$systems 